\title{
Preliminary Studies to Develop a Ubiquitous Computing and Health-monitoring System for Wheelchair Users
}

\author{
Jongbae Kim \\ Dept. of Rehabilitation Science \& Tech. \\ University of Pittsburgh \\ 412-586-6909 \\ jbkim@pitt.edu
}

\author{
Sangyeun Cho \\ Dept. of Computer Science \\ University of Pittsburgh \\ 412-383-7018 \\ cho@cs.pitt.edu
}

\author{
Seung-Jae Kim \\ Center for Adaptive Neural Systems \\ Arizona State University \\ 480-965-9489 \\ Seung-Jae.Kim@asu.edu
}

\begin{abstract}
Both the percentage and raw number of people in need of wheelchairs are increasing every year. Unfortunately, wheelchair users are much more limited in access to conventional IT resources such as home computers and Internet, than their non-disabled counterparts. Moreover, no computer systems specifically designed and optimized for wheelchair users exist today that match the powerful desktop PC systems.

In this research, we envision a wheelchair-worn computing system that is as effectively usable by wheelchair users as other conventional high-performance PC systemsfor general users. We call the system Ubiquitous Computing and Health-monitoring System for Wheelchair Users (u-CHS). As a preliminary study for the development of the u-CHS, we surveyed the needs and usages of the computing and health-monitoring system for wheelchair users in order to fully identify and understand the design requirements for the u-CHS. We received 97 enthusiastic responses from wheelchair users via a voluntary online survey and developed some key design concepts about the input/output, CPU, and health-monitoring modules.
\end{abstract}

\section{Keywords}

Wearable Computer, Ubiquitous Computing, Health-Monitoring, Wheelchair Users, Assistive Technology.

\section{INTRODUCTION}

The number of people in need of power wheelchairs increases every year. In the U.S. alone, an estimated 2.2 million people currently use wheelchairs for their daily mobility [1]. It has been reported, however, that people with disabilities are less than half as likely as their non-disabled counterparts to have access to a computer at home (23.9\% vs. $51.7 \%)$. [2].

Today, unfortunately, no computer systems exist which are specifically designed and optimized for power wheelchair users and also match the powerful desktop PC systems. To remedy this situation, we propose developing a computing system that can be worn on the wheelchair. For a person with a disability who requires a wheelchair for mobility, the wheelchair becomes an extension of the person's body. Therefore this system would be a kind of wearable computing system. It will allow people to independently perform valued and necessary activities so that they can fully participate in society. The ultimate economic benefit is to have more people educated, gainfully employed, and contributing to the tax and consumer bases.

\section{SURVEY STUDY}

We designed a surveyto determine the needs of wheelchair users who would anticipate using the u-CHS on a daily basis. Our aim was to find out how they would operate the system and for what tasks they would use the system. For our survey, we categorized the questions into seven categories: the wheelchair users' current physical condition; work status; educational backgropund; type of wheelchair; health service related questions; computer usage; and recommendation about the design.

105 persons visited our on-line survey web page and we received 97 complete responses over a 44-day period. Most responders were very enthusiastic to answer the questionnaire. $84 \%$ of responders were people with spinal cord injury (SCI) because we contacted the internet communities which are popular to people with SCI. $41 \%$ were using a manual wheelchair, $74 \%$ power wheelchair, and $15 \%$ both of manual and power wheelchair. One of four responders had a tray on the wheelchair.

As for the health-monitoring issues, $81 \%$ of responders currently see a doctor more than once a year, $51 \%$ at least every three months, and $27 \%$ more than once a month. The greatest number of responders wanted to monitor their Blood Pressure (68\%) and Weight (65\%) followed by Blood Sugar (36\%), Pulse (33\%), Temperature (33\%), Blood Oxygen (23\%), Peak Flow (21\%), Fluid Status(19\%), and ECG(11\%).

$33 \%$ carried a laptop when they traveled and $47 \%$ have used the laptop on the wheelchair. For the question of "Which of the following are obstacles that you face when trying to use the laptop from your wheelchair?", their responses were ranked as follows:

1. To plug in the power connection (79\%)

2. To take out the laptop from your backpack or storage area $(71 \%)$

3. To access the table (63\%)

4. To deploy and open the computer (58\%)

5. To use the pointing device (56\%)

6. Plug in the internet connection (45\%)

7. To use the keyboard (40\%)

8. To adjust the monitor angle and position (34\%)

9. To turn on the computer (29\%)

We asked the participants what features must be considered a top priority for the development of this system. We can list the features in the order of highest response as follows:

1. Ease to retrieve (73\%)

2. Convenient Input device (73\%)

3. Durableness/Toughness (69\%)

4. Price $(65 \%)$

5. Outdoor usage (53\%)

6. Ease to hide (52\%)

7. Powerful computing ability (51\%)

8. Convenient Output device (45\%)

9. Environmental protection (41\%)

Permission to make digital or hard copies of all or part of this work for personal or classroom use is granted without fee provided that copies are not made or distributed for profit or commercial advantage and that copies bear this notice and the full citation on the first page. To copy otherwise, to republish, to post on servers or to redistribute to lists, requires prior specific permission and/or a fee. BodyNets 2008, March 13-15 Tempe, Arizona, USA

Copyright () 2008 ICST 978-963-9799-17-2 DOI 10.4108/ICST.BODYNETS2008.2974 


\section{DESIGN OF THE SYSTEM}

The survey study suggests that our system should have at least the following 5 features: 1 ) Ease of retrieving and hiding the input/output unit; 2) Durable and weather protective; 3) Outdoor usage; 4) Powerful computing ability; 5) Embedded weight scale.

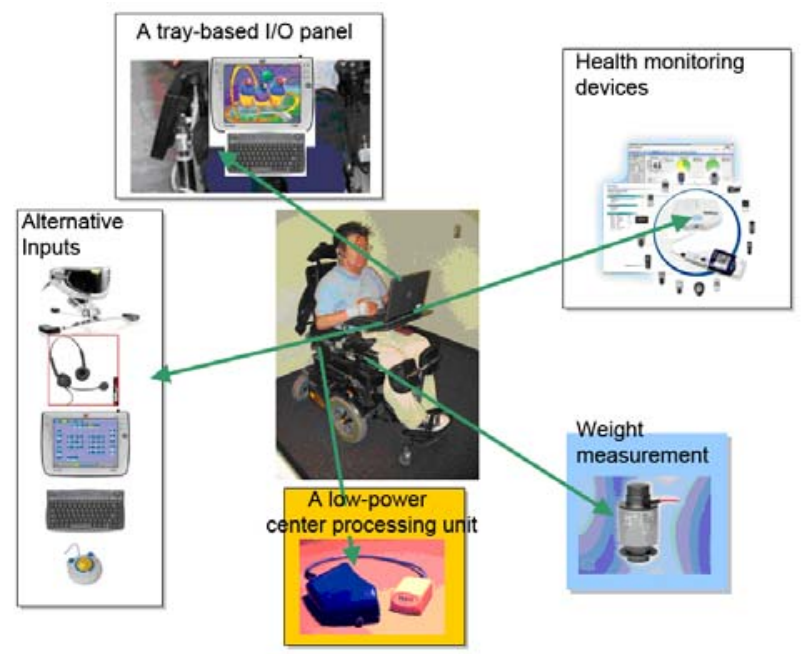

Figure 2. A concept design of the u-CHS for power wheelchair

The deployment of wearable computers is limited by challenges such as weight and battery life. However, for a power wheelchair user, some of these challenges can be mitigated. A power wheelchair user is able to carry a heavier load than an ambulatory individual; and has a power supply continuously available. The arena of wearable computers therefore provides an opportunity for wheelchair-users to bethe vanguard of a trend in technology. Wheelchair users have the potential to make more effective use of wearable computer technology in the short term. Based on the results of our survey study and the benefits of power wheelchairs, we have developed the concept design of the u-CHS for the power wheelchair users. The prototype system will comprise of: (1) CPU unit, (2) Input/Output (I/O) unit, and (3) Health-monitoring unit.

The CPU unit will house the power supply, optional battery, the main board (with the CPU, memory, I/O bridge chips and various RF transceivers), and the hard disk drive. Cable connection bays for the I/O unit are provided. It will have to support various wireless standards for connectivity.

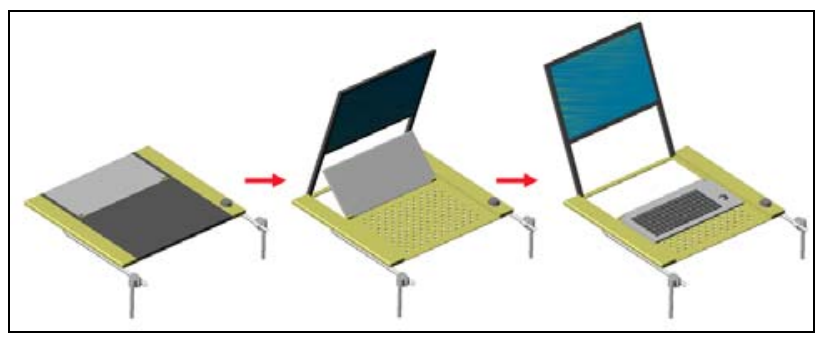

Figure 3. A tray-based I/O panel

Based on our survey results, one of two power wheelchair users keep a tray on their laps, often times because limited function in their hands prevents grasping objects. Therefore, we have designed a tray-based I/O panel, in which a thin LCD display panel and a keyboard/trackball unit are placed on the tray with the back surface upward. They can be deployed and undeployed by activating a switch. It can be used as a normal tray (as in the first frame of Figure 3) when the user does not use the u-CHS.

For wheelchair users who don't use trays, we have designed another style of I/O panel that borrows from the design of pocketretrieval trays located under armrest intypical airplane seats. This Pocket-retrieval I/O panel can be stowed in the armrest of the wheelchair when it is not in use. A great benefit of Pocketretrieval $\mathrm{I} / \mathrm{O}$ panel is that we can apply it also to the u-CHS for manual wheelchair users.

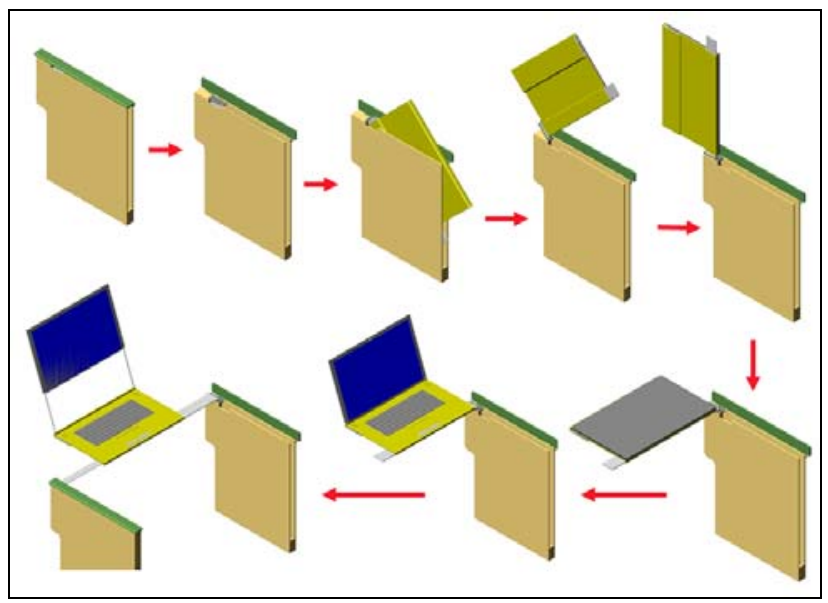

Figure 4. A pocket-retrieval I/O panel

Our survey study also revealedthat wheelchair userswould like to have a way to easily measure their weight (65\% of responders indicated as such). Given their unavoidably sedentary lifestyle - which allows limited opportunities for exercise - and the great difficulty of weighing themselves at home by conventional methods, a novel means of weight monitoring is clearly necessary. Our proposed solution is to put load cells under the seat pan of the wheelchair and integrate a body-weight monitor into the health-monitoring unit of our wheelchair-worn computing system. Not only would this render the problem of weight management more approachable, but subsequently, we suspect it will increase the self-efficacy of many wheelchair-users.

\section{CONCLUSION}

In this study, we have confirmed that the necessity of the Ubiquitous Computing and Health-monitoring System for Wheelchair Users (u-CHS) withsurvey research and also identified the design specifications by designing the concept model of the wheelchair-worn computing system. We believe that the use of the u-CHS will lead to improvement in bothlife satisfaction and the social participation of power wheelchair users.

\section{REFERENCES}

[1] J. McNeil. "Americans With Disabilities: 1997," U.S. Department of Commerce, Economics and Statistics Administration, Bureau of the Census, 2001.

[2] H. Kaye, "Computer and Internet Use Among People with Disabilities. Disability Statistics Report (13),” U.S. Department of Education, National Institute on Disability and Rehabilitation Research. 2003. 